\title{
Factors associated with childhood obesity in Spain. The OBICE study: a case-control study based on sentinel networks
}

\author{
Oscar Zurriaga 1,6,* , Jordi Pérez-Panadés ${ }^{1}$, Joan Quiles Izquierdo ${ }^{1}$, Milagros Gil Costa ${ }^{2}$, \\ Yolanda Anes ${ }^{3}$, Carmen Quiñones ${ }^{4}$, Mario Margolles ${ }^{5}$, Aurora Lopez-Maside ${ }^{1}$, \\ A Tomás Vega-Alonso ${ }^{2}$, María Teresa Miralles Espí and Recent OBICE Research Group \\ ${ }^{1}$ Area de Epidemiologia, Dirección General de Salud Publica, Conselleria de Sanitat, Generalitat Valenciana, \\ Valencia, Spain: ${ }^{2}$ Consejeria de Sanidad, Junta de Castilla y Leon, Valladolid, Spain: ${ }^{3}$ Consejeria de Sanidad y \\ Dependencia, Junta de Extremadura, Merida, Spain: ${ }^{4}$ Consejeria de Salud, Gobierno de La Rioja, Logroño, \\ Spain: ${ }^{5}$ Consejeria de Sanidad, Principado de Asturias, Oviedo, Spain: ${ }^{6}$ Centro Superior de Investigacion en \\ Salud Publica, Avenida de Catalunya 21, 46020-Valencia, Spain
}

Submitted 3 July 2010: Accepted 8 December 2010: First published online 7 February 2011

\begin{abstract}
Objective: To estimate the association strength of dietary behaviour and sedentary habits in relation to childhood obesity in Spain.

Design: A matched case-control study was carried out using data collected by sentinel network paediatricians in general practices.

Setting: Five Spanish autonomous communities.

Subjects: Cases were 437 children (2-14 years old) with BMI $>95$ th percentile according to Spanish reference tables. Controls were 751 children (2-14 years old; two paired per case) with BMI <84th percentile. Data were collected in two phases: individual (questionnaires filled in by sentinel paediatricians) and family (self-administered questionnaires filled in a family environment). Crude OR and adjusted OR (ORc and adj OR) for the given variables were calculated using a simple and multiple conditional logistic regression analysis.

Results: The factors with the greatest effect on obesity were family history of obesity: both parents $(\operatorname{adj} \mathrm{OR}=11 \cdot 2)$, mother but not father (adj OR $=9 \cdot 1)$, father but not mother $(\operatorname{adj} \mathrm{OR}=6 \cdot 1)$, siblings $(\operatorname{adj} \mathrm{OR}=2 \cdot 7)$; and eating between meals (adj $\mathrm{OR}=2 \cdot 5$ ) and consumption of sweets and soft drinks $>2$ times/week (adj $\mathrm{OR}=2 \cdot 0$ ). The highest protection effect was found for five meals per day $(\operatorname{adj} \mathrm{OR}=0 \cdot 5)$, the regular consumption of breakfast (adj OR $=0.5)$ and for eating fruit for dessert (adj OR $=0 \cdot 6$ ). Factors related to sedentary habits did not appear as noteworthy.

Conclusions: We have determined the association between certain dietary behaviour and family history with childhood obesity in several Spanish regions.
\end{abstract}

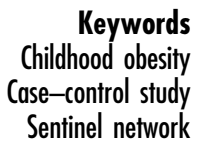

Childhood obesity is determined by a complex interplay of genetic, environmental, behavioural and cultural factors, which lead to an energy imbalance. Social and cultural factors appear to play an important role in shaping the closest behavioural patterns that give rise to body weight gain ${ }^{(1)}$.

In Spain, as well as in other developed countries, the prevalence of childhood obesity has increased in recent decades. From 1984 to 2000, the prevalence in children aged 6-12 years has multiplied by three ${ }^{(2)}$. The highest increase was observed in 10-year-old boys ${ }^{(3)}$.

There have been several cross-sectional studies carried out in Spain on childhood obesity that have allowed us to know the prevalence and its related factors, but there are few case-control design studies to determine factors with association $^{(4-6)}$. One of them ${ }^{(7)}$ suggests that physical leisure-time activity, a family history of obesity, watching television (TV) and sugar-sweetened beverage consumption are important predictive variables for childhood obesity.

None of these case-control studies was carried out using primary health-care cases, but rather with hospital cases. Primary health care in Spain is a proxy of the general population, mainly in childhood, because of its proximity and coverage. Health sentinel networks have been working in the primary health-care level in Spain for $>20$ years $^{(8)}$, and they have proved the effectiveness of carrying out epidemiological studies with different designs in a quick and inexpensive way, because the data are collected during the standard consultation time.

Because childhood obesity is an important adult obesity predictor and a rising problem, we considered that it 
would be interesting to develop the possibility of using this easier way of studying with a case-control design for this topic.

The objective of the present study was to determine the association between dietetic behaviour, physical activity and obesity in children under 15 years of age in a large part of the Spanish population using sentinel networks with an age- and gender-matched case-control study design.

\section{Methods}

The OBICE (OBesidad Infantil en redes CEntinelas) study is a case-control study of childhood obesity and its determining factors in several Spanish regions (autonomous communities) carried out in sentinel networks.

The study population was recruited from 106 paediatric consultations in the sentinel networks of five regions (Asturias, Castilla y Leon, Extremadura, La Rioja and Comunitat Valenciana). These territories cover a population of 1108517 children (2-14 years of age; $20.4 \%$ of the Spanish child population).

Children between 2 and 14 years of age attending a sentinel paediatric consultation (independent of the cause of consultation) were candidates considered eligible for inclusion in the present study. Cases were defined as children with a BMI $>95$ th percentile (according to 'Fundación Orbegozo-Sobradillo' tables ${ }^{(9)}$ ) identified for the first time. Controls (two per case) were children with a BMI <84th percentile (according to the same reference tables) matched to the gender and age ( \pm 1 year) of cases. Sometimes it was not possible to obtain two controls per case, and then only one control was chosen. The study included 1188 individuals, 437 cases and 751 controls. Data were collected in 2007 and 2008.

Children with a pathology that could condition dietary habits and/or physical activity and/or weight and height development were excluded as cases. For controls, obesity as the cause of consultation and a prior diagnosis of obesity (controlled or treated by therapeutic or intervention procedures) were also causes for exclusion. The siblings of cases were excluded as controls. Parents or legal tutors provided consent for both cases and controls.

Two questionnaires were used: one to be filled in by each paediatrician in the practice and another to be filled in by the children's family (self-completed). Both included questions about food frequency.

The paediatrician's questionnaire included information about sex, age, weight and height, country of origin (for children and parents), family background (parental obesity and sibling obesity), personal background (breast-feeding, birth weight and height), physical activity (h/week), screen activities (time use) and dietary habits (breakfast, number of meals per day, whether fruit and vegetables are usually consumed, consumption of sweets and soft drinks per week).
The family's questionnaire also included information about parents' occupation and their educational level, children's sleeping hours, leisure-time physical activity, time spent watching TV, playing video games and using computer (h/week). A semi-quantitative FFQ was integrated into it, including breakfast composition, portions of several foods consumed per week, usual dessert and usual drink and place of the principal meal (school, parents' home or grandparents' home). The questions about food frequency are adapted as a short questionnaire from a validated questionnaire used in other studies ${ }^{(10)}$ in 2005. For the analysis, the food frequency answers were categorized according to the recommendations of the Spanish Society of Community Nutrition ${ }^{(11)}$, and the inadequate consumption by food group was calculated by comparison with Spanish dietary recommendations ${ }^{(12)}$.

\section{Statistical analysis}

Data from both questionnaires were used. Means and 95\% CI for continuous variables and frequencies and percentages for categorical variables were calculated. When the measurement was in h/week, variables were categorized into two categories, indicating risk of obesity or not. A new category named 'not available' was created in some variables whenever data were incomplete in order to improve the power of the analysis. Social class was calculated through both parents' occupations and the family's social class was assigned according to the correspondence analysis method ${ }^{(13)}$. Differences of proportions between cases and controls for each categorical variable were analysed by simple conditional logistic regression $^{(14)}$. The $P$ values of the likelihood ratio test were used. Crude OR (ORc) and 95\% CI were also drawn from simple conditional logistic regression. As we intended to control the potential confounding effect of some of the variables, to estimate the simultaneous effects of multiple variables on the risk of childhood obesity, a multiple conditional logistic regression model with a forward stepwise selection method was performed. The $\chi^{2}$ statistic score was used to assess the importance of each factor at each stepwise run. The statistical significance level required for inclusion was set at $0 \cdot 10$ and for those remaining in the model at $0 \cdot 05$. The adjusted OR (adj OR) was estimated with $95 \%$ CI. Statistical analysis was performed using R statistical software ${ }^{(15)}$ version $2 \cdot 10 \cdot 1$ (R Foundation for Statistical Computing, Vienna, Austria).

\section{Results}

Data from 1188 children were collected. The distribution between sentinel networks was proportional to their population. For 123 cases, two controls could not be obtained.

Table 1 shows a brief description of the principal variables used for matching, as well as the birth weight (g) and height $(\mathrm{cm})$ and the BMI standard deviation score 
Table 1 Characteristics of cases and controls for matching or continuous variables: OBICE study, Spain, 2007-2008

\begin{tabular}{|c|c|c|c|c|}
\hline \multirow[b]{2}{*}{ Variables } & \multicolumn{2}{|c|}{ Cases ( $n$ 437) } & \multicolumn{2}{|c|}{ Controls ( $n 751$ ) } \\
\hline & Mean & $95 \% \mathrm{Cl}$ & Mean & $95 \% \mathrm{Cl}$ \\
\hline Age (years) & $8 \cdot 8$ & $8 \cdot 6,9 \cdot 2$ & $8 \cdot 8$ & $8 \cdot 6,9 \cdot 1$ \\
\hline $\operatorname{Sex}(\%)$ & & & & \\
\hline Boys & \multirow{2}{*}{\multicolumn{2}{|c|}{$\begin{array}{l}55 \cdot 6 \\
44 \cdot 3\end{array}$}} & \multirow{2}{*}{\multicolumn{2}{|c|}{$\begin{array}{l}55 \cdot 5 \\
44 \cdot 4\end{array}$}} \\
\hline Girls & & & & \\
\hline BMI-SDS (British 1990 reference) & $2 \cdot 773$ & $2 \cdot 729,2 \cdot 817$ & 0.207 & $0.138,0.275$ \\
\hline Birth weight $(\mathrm{g})$ & 3289 & 3239,3341 & 3191 & 3155,3229 \\
\hline Height at birth (cm) & $50 \cdot 0$ & $49 \cdot 8,50 \cdot 2$ & $49 \cdot 8$ & $49 \cdot 6,50 \cdot 0$ \\
\hline
\end{tabular}

OBICE, OBesidad Infantil en redes CEntinelas; BMI-SDS, standard deviation score BMI.

(BMI-SDS; BMI was converted into SDS using the revised British 1990 reference) ${ }^{(16,17)}$. According to international standards criteria, we identified from our defined cases eighty-seven children as non-obese, but all of them were overweight according to the same criteria. For controls, there were ninety children with overweight according to international standards criteria but none of them were obese according to the same criteria.

The distribution for variables included in the paediatrician's questionnaire, their ORc, the respective 95\% CI and statistical significance test are presented in Table 2. In the crude analysis (non-adjusted), cases had a higher proportion of history of family obesity (parents or siblings) than did controls. Other identified obesity risk factors were the consumption of sweets and soft drinks $>2$ times/week, $>3 \mathrm{~h} / \mathrm{d}$ of screen activities and eating between meals.

In the crude analysis, dietary variables with a protection value and statistical differences between cases and controls were: consuming five meals per day, daily consumption of fruit, the usual consumption of vegetables, usual school refectory use, $>2$ h of leisure-time physical activity, regular consumption of breakfast, consuming fruit for dessert, a healthy breakfast, sleeping $\geq 10 \mathrm{~h}$ and not using soft drinks in meals as usual. The family's social class showed a $P$ value equal to 0.005 with a difference between low (reference) and high family social class $(\mathrm{ORc}=0 \cdot 4)$. The parents' educational level (secondary education or more) showed an ORc of 0.7 for the mother's education and 0.6 for the father's education.

Table 3 shows the results of the semi-quantitative FFQ integrated into the family's questionnaire (ORc, 95\% CI and $P$ values). In the crude analysis, some of the dietary factors showed a statistically significant difference $(P<0 \cdot 001)$ between cases and controls. They were: recommended consumption of fish and a consumption of soft drinks $<2$ times/week.

Table 4 shows the result of the simultaneous effects of multiple variables on the risk of childhood obesity through the multiple conditional logistic regression analysis (adj OR, 95\% CI and $P$ value). The factors with more effect on childhood obesity were those included in the family's history of obesity. The highest effect was for both parents' obesity: when both parents were obese the adj OR was $11 \cdot 2$. If the father was obese, but not the mother, the adj OR was $6 \cdot 12$. If the mother was obese, but not the father, the adj OR was 9·08. Siblings' obesity showed an adj OR close to $3 \cdot 0$. The dietary behaviour with a greater strength of association with obesity was snacking or eating between meals (adj $\mathrm{OR}=2 \cdot 5$ ), followed by the consumption of sweets and soft drinks $>2$ times/week (adj $\mathrm{OR}=2 \cdot 0$ ). The highest protection effect was found for consumption of five meals per day (adj OR $=0.5$ ), regular consumption of breakfast $(\operatorname{adj} \mathrm{OR}=0.5)$ and consuming fruit for dessert $(\operatorname{adj} \mathrm{OR}=0 \cdot 6)$. The other factor included in the final model was the consumption of meat, but the $95 \%$ CI of the adj OR for more or less consumption was not significant.

\section{Discussion}

The main results of the OBICE study showed the importance of the family environment in several parts of Spain, especially parents' obesity, as a risk factor for childhood obesity. The results are not representative for the Spanish child population, but the regions included represent an important proportion of Spanish children (20.4\%). As far as we know, the present study is the first non-hospitalbased case-control study on childhood obesity in Spain with a wide population framework, both geographically and proportionally.

The selection of cut-off points for obesity excluded the overlap between cases and controls. We chose a BMI value of $>95$ th percentile for considering a child as obese because this was the criterion recommended and frequently used in epidemiological studies on childhood obesity $^{(18-20)}$. Subsequent recommendations ${ }^{(3)}$ indicate the use of the 97th percentile. Thus, it is possible that our case population includes individuals who could be categorized as overweight, meaning that the results underestimate some of the real effects of obesity; however, it is known $^{(21)}$ that the most frequently used Spanish tables (the Hernández tables ${ }^{(22)}$ ) overestimate obesity, hence the effect would be minor. Moreover, the quantity of cases between the 95th and 97th percentiles in our study 
Table 2 Risk factors for childhood obesity from paediatrician's and family's questionnaires: OBICE study, Spain, 2007-2008

\begin{tabular}{|c|c|c|c|c|c|c|c|}
\hline \multirow[b]{2}{*}{ Risk factors } & \multicolumn{2}{|c|}{ Cases $(n 437)$} & \multicolumn{2}{|c|}{ Controls $(n 751)$} & \multirow[b]{2}{*}{ Crude OR } & \multirow[b]{2}{*}{$95 \% \mathrm{Cl}$} & \multirow[b]{2}{*}{$P$ value ${ }^{*}$} \\
\hline & $n$ & $\%$ & $n$ & $\%$ & & & \\
\hline Parental obesity & & & & & & & $<0.001$ \\
\hline Father non-obese/mother non-obese (ref.) & 176 & $40 \cdot 3$ & 623 & $83 \cdot 0$ & & & \\
\hline Father obese/mother non-obese & 97 & $22 \cdot 2$ & 63 & $8 \cdot 4$ & $6 \cdot 5$ & $4 \cdot 2,10 \cdot 0$ & \\
\hline Father non-obese/mother obese & 86 & $19 \cdot 7$ & 40 & $5 \cdot 3$ & $9 \cdot 5$ & $5 \cdot 8,15 \cdot 5$ & \\
\hline Father obese/mother obese & 78 & $17 \cdot 9$ & 25 & $3 \cdot 3$ & $12 \cdot 1$ & $6 \cdot 9,21 \cdot 2$ & \\
\hline Siblings' obesity & & & & & & & $<0.001$ \\
\hline No (ref.) & 144 & $32 \cdot 9$ & 334 & $44 \cdot 4$ & & & \\
\hline Yes & 88 & $20 \cdot 1$ & 39 & $5 \cdot 1$ & $5 \cdot 4$ & $3 \cdot 4,8 \cdot 8$ & \\
\hline Not available & 205 & $46 \cdot 9$ & 378 & $50 \cdot 3$ & $1 \cdot 3$ & $0.9,1.8$ & \\
\hline Breast-feeding & & & & & & & 0.046 \\
\hline No (ref.) & 70 & $16 \cdot 0$ & 114 & $15 \cdot 1$ & & & \\
\hline Yes & 302 & $69 \cdot 1$ & 556 & $74 \cdot 0$ & $0 \cdot 8$ & $0 \cdot 6,1 \cdot 2$ & \\
\hline Not available & 65 & $14 \cdot 8$ & 81 & $10 \cdot 7$ & $1 \cdot 4$ & $0.8,2.5$ & \\
\hline Five meals per day & & & & & & & $<0.001$ \\
\hline No (ref.) & 140 & $32 \cdot 0$ & 147 & $19 \cdot 5$ & & & \\
\hline Yes & 297 & $67 \cdot 9$ & 604 & $80 \cdot 4$ & 0.4 & $0.3,0.6$ & \\
\hline Daily consumption of fruit & & & & & & & $<0.001$ \\
\hline No (ref.) & 250 & $57 \cdot 2$ & 333 & $44 \cdot 3$ & & & \\
\hline Yes & 187 & $42 \cdot \overline{7}$ & 418 & $55 \cdot 6$ & 0.5 & $0 \cdot 4,0 \cdot 7$ & \\
\hline Usual consumption of vegetables & & & & & & & $<0.001$ \\
\hline No (ref.) & 230 & $52 \cdot 6$ & 308 & $41 \cdot 0$ & & & \\
\hline Yes & 207 & $47 \cdot 3$ & 443 & $58 \cdot 9$ & $0 \cdot 6$ & $0.4,0.7$ & \\
\hline Sweets and soft drinks consumption & & & & & & & $<0.001$ \\
\hline$\leq 2$ times/week (ref.) & 195 & $44 \cdot 6$ & 510 & $67 \cdot 9$ & & & \\
\hline$>2$ times/week & 224 & $51 \cdot 2$ & 221 & $29 \cdot 4$ & $2 \cdot 8$ & $2 \cdot 1,3 \cdot 7$ & \\
\hline Not available & 18 & $4 \cdot 1$ & 20 & $2 \cdot 6$ & $2 \cdot 1$ & $1 \cdot 0,4 \cdot 4$ & \\
\hline Usual school refectory use & & & & & & & 0.025 \\
\hline No (ref.) & 331 & $75 \cdot 7$ & 531 & $70 \cdot 7$ & & & \\
\hline Yes & 104 & $23 \cdot 8$ & 219 & $29 \cdot 1$ & $0 \cdot 6$ & $0.5,0.9$ & \\
\hline Not available & 2 & 0.4 & 1 & 0.1 & $3 \cdot 7$ & $0 \cdot 3,41 \cdot 8$ & \\
\hline Screen hours (TV + computer) & & & & & & & $<0.001$ \\
\hline$<3 \mathrm{~h} / \mathrm{d}$ (ref.) & 230 & $52 \cdot 6$ & 479 & $63 \cdot 7$ & & & \\
\hline$\geq 3 \mathrm{~h} / \mathrm{d}$ & 182 & $41 \cdot 6$ & 237 & $31 \cdot 5$ & $1 \cdot 8$ & $1 \cdot 3,2 \cdot 4$ & \\
\hline Not available & 25 & $5 \cdot 7$ & 35 & $4 \cdot 6$ & 1.5 & $0.9,2.7$ & \\
\hline Leisure-time physical activity (sport) & & & & & & & $<0.001$ \\
\hline$<2 \mathrm{~h} /$ week (ref.) & 246 & $56 \cdot 2$ & 339 & $45 \cdot 1$ & & & \\
\hline$\geq 2 \mathrm{~h} /$ week & 187 & $42 \cdot \overline{7}$ & 404 & $53 \cdot 7$ & $0 \cdot 6$ & $0 \cdot 4,0.7$ & \\
\hline Not available & 4 & 0.9 & 8 & $1 \cdot 0$ & $0 \cdot 6$ & $0 \cdot 1,2 \cdot 5$ & \\
\hline Regular consumption of breakfast & & & & & & & $<0.001$ \\
\hline No (ref.) & 46 & $10 \cdot 5$ & 37 & 4.9 & & & \\
\hline Yes & 391 & $89 \cdot 4$ & 714 & $95 \cdot 0$ & $0 \cdot 3$ & $0.2,0.6$ & \\
\hline Mother's educational level & & & & & & & 0.084 \\
\hline Below secondary education (ref.) & 251 & $57 \cdot 4$ & 380 & $50 \cdot 6$ & & & \\
\hline Secondary education or above & 182 & $41 \cdot 6$ & 365 & $48 \cdot 6$ & $0 \cdot 7$ & $0.5,0.9$ & \\
\hline Not available & 4 & 0.9 & 6 & 0.8 & $1 \cdot 0$ & $0 \cdot 2,3 \cdot 7$ & \\
\hline Father's educational level & & & & & & & 0.008 \\
\hline Below secondary education (ref.) & 251 & $57 \cdot 4$ & 367 & $48 \cdot 8$ & & & \\
\hline Secondary education or above & 165 & $37 \cdot 7$ & 349 & $46 \cdot 4$ & $0 \cdot 6$ & $0.5,0.8$ & \\
\hline Not available & 21 & $4 \cdot 8$ & 35 & $4 \cdot 6$ & $0 \cdot 8$ & $0.4,1 \cdot 4$ & \\
\hline Family's social class & & & & & & & 0.005 \\
\hline Low (ref.) & 82 & $18 \cdot 7$ & 118 & $15 \cdot 7$ & & & \\
\hline Medium & 290 & $66 \cdot 3$ & 483 & $64 \cdot 3$ & $0 \cdot 8$ & $0 \cdot 6,1 \cdot 2$ & \\
\hline High & 35 & $8 \cdot 0$ & 112 & $14 \cdot 9$ & $0 \cdot 4$ & $0 \cdot 2,0 \cdot 7$ & \\
\hline Not available & 30 & $6 \cdot 8$ & 38 & $5 \cdot 0$ & $1 \cdot 1$ & $0 \cdot 6,2 \cdot 0$ & \\
\hline Eating between meals & & & & & & & $<0.001$ \\
\hline No (ref.) & 245 & $56 \cdot 0$ & 594 & $79 \cdot 0$ & & & \\
\hline Yes & 192 & 43.9 & 157 & $20 \cdot 9$ & $3 \cdot 0$ & $2 \cdot 3,4 \cdot 0$ & \\
\hline Consuming fruit for dessert & & & & & & & $<0.001$ \\
\hline No (ref.) & 307 & $70 \cdot 2$ & 441 & $58 \cdot 7$ & & & \\
\hline Yes & 127 & $29 \cdot 0$ & 306 & $40 \cdot 7$ & 0.5 & $0 \cdot 4,0 \cdot 7$ & \\
\hline Not available & 3 & 0.6 & 4 & 0.5 & $1 \cdot 2$ & $0 \cdot 2,5 \cdot 5$ & \\
\hline Healthy breakfast & & & & & & & 0.007 \\
\hline No (ref.) & 412 & $94 \cdot 2$ & 677 & $90 \cdot 1$ & & & \\
\hline Yes (milk + juice + corn flakes, bread or cookies) & 25 & $5 \cdot 7$ & 74 & $9 \cdot 8$ & 0.5 & $0.3,0.8$ & \\
\hline Sleep & & & & & & & 0.009 \\
\hline$<10 \mathrm{~h}$ (ref.) & 229 & $52 \cdot 4$ & 333 & $44 \cdot 3$ & & & \\
\hline$\geq 10 \mathrm{~h}$ & 204 & $46 \cdot 6$ & 411 & $54 \cdot 7$ & $0 \cdot 6$ & $0.4,0.8$ & \\
\hline Not available & 4 & 0.9 & 7 & 0.9 & $0 \cdot 7$ & $0.2,2.5$ & \\
\hline Usual drink for meals & & & & & & & $<0.001$ \\
\hline Soft drinks (ref.) & 73 & $16 \cdot 7$ & 61 & $8 \cdot 1$ & & & \\
\hline Non-soft drinks & $357 \cdot 0$ & $81 \cdot 6$ & 663 & $88 \cdot 2$ & 0.4 & $0.3,0.6$ & \\
\hline Not available & 7 & $1 \cdot 6$ & 27 & $3 \cdot 6$ & $0 \cdot 2$ & $0.0,0.5$ & \\
\hline
\end{tabular}

OBICE, OBesidad Infantil en redes CEntinelas; ref., reference category; TV, television.

Results of the simple conditional logistic regression analysis of risk factors for childhood obesity are presented here.

*Likelihood ratio test. 
Table 3 Risk factors for childhood obesity from family's questionnaire (semi-quantitative FFQ): OBICE study, Spain, 2007-2008

\begin{tabular}{|c|c|c|c|c|c|c|c|}
\hline \multirow[b]{2}{*}{ Risk factors } & \multicolumn{2}{|c|}{ Cases ( $n$ 437) } & \multicolumn{2}{|c|}{ Controls ( $n$ 751) } & \multirow[b]{2}{*}{ Crude OR } & \multirow[b]{2}{*}{$95 \% \mathrm{Cl}$} & \multirow[b]{2}{*}{$P$ value } \\
\hline & $n$ & $\%$ & $n$ & $\%$ & & & \\
\hline Consumption of dairy products & & & & & & & 0.014 \\
\hline Not daily (ref.) & 37 & $8 \cdot 4$ & 39 & $5 \cdot 1$ & & & \\
\hline Daily & 396 & $90 \cdot 6$ & 710 & $94 \cdot 5$ & 0.5 & $0 \cdot 3,0 \cdot 8$ & \\
\hline Not available & 4 & 0.9 & 2 & $0 \cdot 2$ & $2 \cdot 2$ & $0 \cdot 4,12 \cdot 8$ & \\
\hline Consumption of meat & & & & & & & 0.091 \\
\hline >3 times/week (ref.) & 342 & $78 \cdot 2$ & 626 & $83 \cdot 3$ & & & \\
\hline$<2$ times/week & 89 & $20 \cdot \overline{3}$ & 120 & $15 \cdot 9$ & $1 \cdot 3$ & $0 \cdot 9,1 \cdot 8$ & \\
\hline Not available & 6 & $1 \cdot 3$ & 5 & $0 \cdot 6$ & $2 \cdot 3$ & $0 \cdot 7,7 \cdot 9$ & \\
\hline Consumption of cold meat & & & & & & & 0.972 \\
\hline Daily (ref.) & 107 & $24 \cdot 4$ & 186 & $24 \cdot 7$ & & & \\
\hline Not daily & 325 & $74 \cdot 3$ & 557 & $74 \cdot 1$ & $1 \cdot 0$ & $0 \cdot 7,1 \cdot 3$ & \\
\hline Not available & 5 & $1 \cdot 1$ & 8 & $1 \cdot 0$ & $1 \cdot 0$ & $0 \cdot 3,3 \cdot 6$ & \\
\hline Consumption of fish & & & & & & & $<0.001$ \\
\hline$<1$ time/week (ref.) & 126 & $28 \cdot 8$ & 141 & $18 \cdot 7$ & & & \\
\hline$\geq 1$ time/week & 309 & $70 \cdot 7$ & 609 & $81 \cdot 0$ & 0.5 & $0 \cdot 4,0 \cdot 7$ & \\
\hline Not available & 2 & $0 \cdot 4$ & 1 & $0 \cdot 1$ & $2 \cdot 2$ & $0 \cdot 1,26 \cdot 6$ & \\
\hline Consumption of eggs & & & & & & & 0.956 \\
\hline Daily (ref.) & 16 & $3 \cdot 6$ & 27 & $3 \cdot 6$ & & & \\
\hline Not daily & 420 & $96 \cdot 1$ & 722 & $96 \cdot 1$ & $1 \cdot 0$ & $0 \cdot 5,2 \cdot 0$ & \\
\hline Not available & 1 & $0 \cdot 2$ & 2 & $0 \cdot 2$ & $0 \cdot 8$ & $0.0,9 \cdot 9$ & \\
\hline Consumption of vegetables & & & & & & & 0.502 \\
\hline Not daily (ref.) & 341 & $78 \cdot 0$ & 587 & $78 \cdot 1$ & & & \\
\hline Daily & 92 & $21 \cdot 0$ & 161 & $21 \cdot 4$ & 0.9 & $0 \cdot 7,1 \cdot 3$ & \\
\hline Not available & 4 & 0.9 & 3 & $0 \cdot 4$ & $2 \cdot 4$ & $0.5,10 \cdot 9$ & \\
\hline Consumption of fruit & & & & & & & 0.006 \\
\hline Not daily (ref.) & 197 & $45 \cdot 0$ & 284 & $37 \cdot 8$ & & & \\
\hline Daily & 234 & $53 \cdot 5$ & 463 & $61 \cdot 6$ & $0 \cdot 7$ & $0.5,0.9$ & \\
\hline Not available & 6 & $1 \cdot 3$ & 4 & 0.5 & $2 \cdot 3$ & $0 \cdot 6,8 \cdot 3$ & \\
\hline Consumption of bread & & & & & & & 0.414 \\
\hline Not daily (ref.) & 73 & $16 \cdot 7$ & 104 & $13 \cdot 8$ & & & \\
\hline Daily & 362 & $82 \cdot 8$ & 643 & $85 \cdot 6$ & $0 \cdot 8$ & $0 \cdot 5,1 \cdot 1$ & \\
\hline Not available & 2 & $0 \cdot 4$ & 4 & 0.5 & $0 \cdot 8$ & $0 \cdot 1,4 \cdot 6$ & \\
\hline Consumption of rice, potatoes & & & & & & & 0.375 \\
\hline 3 times/week (ref.) & 9 & $2 \cdot 0$ & 8 & $1 \cdot 0$ & & & \\
\hline$<3$ times/week & 427 & $97 \cdot 7$ & 739 & $98 \cdot 4$ & 0.5 & $0 \cdot 1,1 \cdot 4$ & \\
\hline Not available & 1 & $0 \cdot 2$ & 4 & 0.5 & $0 \cdot 2$ & $0 \cdot 0,2 \cdot 9$ & \\
\hline Consumption of fried foods & & & & & & & $0 \cdot 419$ \\
\hline$>3$ times/week (ref.) & 112 & $25 \cdot 6$ & 170 & $22 \cdot 6$ & & & \\
\hline$<2$ times/week & 321 & $73 \cdot 4$ & 574 & $76 \cdot 4$ & $0 \cdot 8$ & $0 \cdot 6,1 \cdot 1$ & \\
\hline Not available & 4 & 0.9 & 7 & 0.9 & $0 \cdot 8$ & $0 \cdot 2,3 \cdot 0$ & \\
\hline Consumption of pulses & & & & & & & 0.053 \\
\hline$<1$ time/week (ref.) & 66 & $15 \cdot 1$ & 84 & $11 \cdot 1$ & & & \\
\hline$\geq 1$ time/week & 369 & $84 \cdot 4$ & 656 & $87 \cdot 3$ & $0 \cdot 7$ & $0 \cdot 5,1 \cdot 0$ & \\
\hline Not available & 2 & 0.4 & 11 & $1 \cdot 4$ & $0 \cdot 2$ & $0 \cdot 0,1 \cdot 1$ & \\
\hline Consumption of pre-cooked meals & & & & & & & $0 \cdot 251$ \\
\hline$\geq 1$ time/week (ref.) & 133 & $30 \cdot 4$ & 209 & $27 \cdot 8$ & & & \\
\hline$<1$ time/week & 299 & $68 \cdot 4$ & 538 & $71 \cdot 6$ & $0 \cdot 8$ & $0 \cdot 6,1 \cdot 1$ & \\
\hline Not available & 5 & $1 \cdot 1$ & 4 & 0.5 & 1.9 & $0 \cdot 5,7 \cdot 4$ & \\
\hline Consumption of snacks & & & & & & & 0.003 \\
\hline >3 times/week (ref.) & 86 & $19 \cdot 6$ & 91 & $12 \cdot 1$ & & & \\
\hline$<2$ times/week & 348 & $79 \cdot 6$ & 653 & $86 \cdot 9$ & 0.5 & $0 \cdot 4,0 \cdot 7$ & \\
\hline Not available & 3 & $0 \cdot 6$ & 7 & 0.9 & $0 \cdot 4$ & $0 \cdot 1,1 \cdot 9$ & \\
\hline Consumption of cake, chocolate & & & & & & & $0 \cdot 745$ \\
\hline$\geq 1$ time/week (ref.) & 226 & $51 \cdot 7$ & 404 & $53 \cdot 7$ & & & \\
\hline$<1$ time/week & 208 & $47 \cdot 6$ & 343 & $45 \cdot 6$ & $1 \cdot 1$ & $0 \cdot 8,1 \cdot 4$ & \\
\hline Not available & 3 & $0 \cdot 6$ & 4 & 0.5 & $1 \cdot 3$ & $0 \cdot 2,6 \cdot 0$ & \\
\hline Consumption of sweets & & & & & & & 0.098 \\
\hline$>3$ times/week (ref.) & 95 & $21 \cdot 7$ & 124 & $16 \cdot 5$ & & & \\
\hline$<2$ times/week & 339 & $77 \cdot 5$ & 622 & $82 \cdot 8$ & $0 \cdot 7$ & $0.5,0.9$ & \\
\hline Not available & 3 & $0 \cdot 6$ & 5 & $0 \cdot 6$ & $0 \cdot 7$ & $0 \cdot 1,3 \cdot 2$ & \\
\hline Consumption of soft drinks & & & & & & & $<0.001$ \\
\hline$>3$ times/week (ref.) & 124 & $28 \cdot 3$ & 105 & $13 \cdot 9$ & & & \\
\hline$<2$ times/week & 310 & $70 \cdot 9$ & 645 & $85 \cdot 8$ & $0 \cdot 3$ & $0.2,0.5$ & \\
\hline Not available & 3 & $0 \cdot 6$ & 1 & $0 \cdot 1$ & $2 \cdot 7$ & $0 \cdot 2,27 \cdot 0$ & \\
\hline
\end{tabular}

OBICE, OBesidad Infantil en redes CEntinelas; ref., reference category.

Results of the simple conditional logistic regression analysis of risk factors for childhood obesity are presented here.

*Likelihood ratio test. 
Table 4 Multiple conditional logistic regression analysis of risk factors for childhood obesity: OBICE study, Spain, 2007-2008

\begin{tabular}{|c|c|c|c|}
\hline Risk factors & Adjusted OR & $95 \% \mathrm{Cl}$ & $P$ value* \\
\hline $\begin{array}{l}\text { Parental obesity } \\
\text { Father non-obese/mother non-obese (ref.) }\end{array}$ & & & $<0.001$ \\
\hline Father obese/mother non-obese & $6 \cdot 1$ & $3 \cdot 8,10 \cdot 0$ & \\
\hline Father non-obese/mother obese & $9 \cdot 1$ & $5 \cdot 1,16 \cdot 3$ & \\
\hline Father obese/mother obese & $11 \cdot 2$ & $5 \cdot 9,21 \cdot 3$ & \\
\hline $\begin{array}{l}\text { Siblings' obesity } \\
\text { No (ref.) }\end{array}$ & & & 0.008 \\
\hline Yes & $2 \cdot 7$ & $1 \cdot 4,5 \cdot 0$ & \\
\hline Not available & $1 \cdot 4$ & $0 \cdot 9,2 \cdot 1$ & \\
\hline $\begin{array}{l}\text { Regular consumption of breakfast } \\
\text { No (ref.) }\end{array}$ & & & 0.042 \\
\hline Yes & 0.5 & $0.26,0.98$ & \\
\hline $\begin{array}{l}\text { Five meals per day } \\
\text { No (ref.) }\end{array}$ & & & 0.002 \\
\hline Yes & 0.5 & $0.3,0.8$ & \\
\hline $\begin{array}{l}\text { Eating between meals } \\
\text { No (ref.) }\end{array}$ & & & $<0.001$ \\
\hline Yes & $2 \cdot 5$ & $1 \cdot 7,3 \cdot 6$ & \\
\hline $\begin{array}{l}\text { Sweets and soft drinks consumption } \\
\leq 2 \text { times/week (ref.) }\end{array}$ & & & $<0.001$ \\
\hline$>2$ times/week & $2 \cdot 0$ & $1 \cdot 4,2 \cdot 9$ & \\
\hline Not available & $2 \cdot 1$ & $0 \cdot 8,5 \cdot 4$ & \\
\hline $\begin{array}{l}\text { Consuming fruit for dessert } \\
\text { No (ref.) }\end{array}$ & & & 0.002 \\
\hline Yes & $0 \cdot 6$ & $0 \cdot 4,0 \cdot 8$ & \\
\hline Not available & $7 \cdot 5$ & $0 \cdot 7,78 \cdot 5$ & \\
\hline $\begin{array}{l}\text { Consumption of meat } \\
>3 \text { times/week (ref.) }\end{array}$ & & & 0.020 \\
\hline$\leq 2$ times/week & $1 \cdot 2$ & $0 \cdot 8,1 \cdot 8$ & \\
\hline Not available & $8 \cdot \overline{5}$ & $2 \cdot 0,36 \cdot 5$ & \\
\hline
\end{tabular}

OBICE, OBesidad Infantil en redes CEntinelas; ref., reference category.

*Likelihood ratio test.

was relatively small. When we repeated the analysis, excluding cases and their associated controls between the 95th and the 97th percentiles (fifty-three cases and ninetysix controls), the results were essentially unchanged (results not shown).

Many studies emphasise the importance of parents' obesity, especially the mother's obesity, as a risk factor for childhood obesity ${ }^{(25-27)}$, showing that parents' obesity and overweight increase the risk of childhood obesity. This is because, in addition to genetic factors, family members share behavioural risk factors including energy and percentage of fat intake, food preferences ${ }^{(28)}$ and physical activity, which may influence children's weight later on. After performing the crude analysis, the final multivariate model excluded potential confounders such as breast-feeding ${ }^{(23)}$, social class ${ }^{(24)}$ and some dietetic factors and physical activity related to parental obesity ${ }^{(19)}$. The final model showed an association with parents' and siblings' obesity, highlighting the importance of obesity prevention in the family environment, involving fathers and mothers actively in acquiring knowledge to adopt healthy behaviour for diet and physical activity, because there is evidence of the long-term effectiveness of familybased treatment programmes for obese children ${ }^{(29)}$.

The categorization as obese for parents and siblings was based on their own information and modified by the paediatrician's observations, but it was very difficult to make a direct measurement in a standard high-pressure health-care environment. Owing to this fact, our results are affected because informants systematically overestimated the height and underestimated the weight of their family members ${ }^{(30,31)}$, underestimating obesity (in approximately one person out of three ${ }^{(32)}$, but the bias could be partially made up for by face-to-face contact, which reduces it ${ }^{(33)}$.

The results showed dietary factors as a main risk, such as eating between meals and the consumption of sweets and soft drinks $>2$ times/week. Although there is little information about meal frequency in children and adolescents, a similar pattern has been described ${ }^{(12)}$ in Spanish children and young people, related to spending more time watching TV ('snacky' pattern). Other stu$\operatorname{dies}^{(34)}$ have also related snacking to the consumption of snacks and soft drinks while watching TV. In the OBICE study, after adjusting screen activity hours (including TV), the factors remain, and snacking seems to be an important factor associated with childhood obesity independently of other behavioural or social factors. One limitation of our results is the lack of knowledge about the composition of this snacking. With regard to soft drink consumption, there is clear evidence ${ }^{(35)}$ about the associations of soft drink consumption with increased energy intake and body weight in children, adolescents and adults ${ }^{(36)}$. Other studies carried out in Spain on 
6-7-year-old children ${ }^{(37)}$ showed that the impact of sweetened soft drinks, together with bakery products and yoghurt, on the quality of their diet is only modest.

The factors inversely associated with childhood obesity found in our study were meal frequency, regular consumption of breakfast and consumption of fruit for dessert. Bellisle et $a l{ }^{(38)}$ have concluded that there does not appear to be a relationship between meal patterning and obesity; however, our results on meal frequency are consistent with those of other studies in children, in which a high meal frequency was inversely associated with childhood obesity $^{(39,40)}$, not explained by potential confounders.

There is a lot of evidence ${ }^{(41)}$ that regularly consuming breakfast is a protective factor and skipping it is associated with childhood, adolescent and pre-school ${ }^{(42)}$ obesity. The percentage skipping breakfast shown by the OBICE results is high $(10 \cdot 53 \%)$ for obesity cases, particularly compared with results in Spanish adolescents presented in the AVENA (Alimentación y Valoración del Estado Nutricional en Adolescentes) Study (8.6\% in females and $3.5 \%$ in males) ${ }^{(43)}$, similar to the enKid study $^{(44)}(8 \cdot 2 \%$ for children and adolescents). To fight against childhood obesity it is necessary to promote breakfast as a main meal (15-20\% energy intake per day).

To consume fruit for dessert appears as an important protective factor. Increased fruit consumption has been associated $^{(45)}$ with a lower risk of a medium weight gain for adults, and the enKid study ${ }^{(44)}$ in Spain has shown that a lower consumption of fruit and vegetables is associated with a higher prevalence of obesity. Regarding the behaviour of consuming fresh fruit for dessert, one of the bases of the Mediterranean diet pattern ${ }^{(46)}$, and the relationship with childhood obesity, there is not enough evidence in the literature.

Factors related to sedentary habits did not appear as noteworthy in the final results, but the univariate analysis showed that controls presented a better situation than cases with regard to time for screen activities and physical activities. It is possible that they could be affected by a non-differential misclassification because of the measurement difficulties for physical activity variables, particularly in the health-care environment.

Previous studies in both children and adults have shown an increased prevalence of obesity associated with lower sleeping duration ${ }^{(47)}$. In our study, the apparent protective effect associated with $\geqslant 10 \mathrm{~h}$ of sleep seen in the univariate analysis was not statistically significant in the multivariate analysis. However, if sleep deprivation causes obesity through its effects on decreasing physical activity and activation of hormonal pathways leading to increasing appetite ${ }^{(48)}$, our multivariate model might be overadjusting for the mechanisms that explain this relationship.

The use of sentinel networks implies strengths and weaknesses for this kind of survey with a case-control design. Its strength is that it is an easy way of conducting the study because the networks are ready to readdress the topic of study and conduct it within a short time; in addition, it is possible to obtain a sample closer to the general population than for other types of approximations. Its weakness is the participation of a lot of researchers with the possibility of different criteria. To avoid this, a study protocol with strict indications was distributed to each participant and the network coordination centres assumed the validation and homogenization task. Another difficulty was the selection of controls because at certain ages healthy non-obese children do not attend paediatric consultations. Therefore, it was not possible to obtain two controls for each case for all cases, but the proportion of cases $(28 \cdot 14 \%)$ with only one control was relatively small. Finally, an important limitation was produced by the already mentioned auto-report of the parents' and sibling's obesity.

The OBICE study has shown the importance of obesity prevention in the family environment and the need to act on certain dietary habits in childhood, increasing the frequency of meals, reducing the consumption of sweets and soft drinks, promoting breakfast and healthy desserts. The results of the OBICE study also show the power of health sentinel networks to implement this type of epidemiological design.

\section{Acknowledgements}

The present study was partially funded by a Spanish Science and Innovation Ministry grant (Fondo de Investigación Sanitaria, Convocatoria de Ayudas Para Proyectos de Investigación del Programa de Promoción de la Investigación Biomédica y en Ciencias de la Salud del Ministerio de Sanidad y Consumo en 2006, Expediente PI06/0923). The authors have no conflict of interest to declare. O.Z. designed the study, participated in the analysis and wrote the first draft. J.P.-P. handled the data analysis and performed the statistical analysis. J.Q.I., M.G.C., Y.A., C.Q., M.M., A.L.-M., A.T.V.-A. and M.T.M.E. collaborated in the design of the study and also participated in the analysis and manuscript development. The RECENT OBICE Research Group participated in collecting the data. The authors thank all the paediatricians and public health professionals involved in the health sentinel networks throughout the autonomous communities of Castilla y León, Extremadura, Asturias, La Rioja and Comunitat Valenciana for their voluntary work. They also acknowledge F. Javier Nieto (University of Wisconsin - Madison, USA) for his useful comments on previous versions of the manuscript, and Carlos Abellan for his useful help.

The RECENT OBICE Research Group comprises: José Amancio Peñuelas Ruiz, María Jesús Redondo Gallego, Alejandro Cremades Bernabeu (Dirección General de Salud Pública, Conselleria de Sanitat, Generalitat Valenciana, Valencia, Spain); Ana María Sacristán Martín, Rufino Álamo Sanz (Consejeria de Sanidad, Junta de Castilla y León, 
Valladolid, Spain); Bertomeu Serra Pons, Arturo Meliveo Moreno (Agencia Valenciana de Salud, Valencia, Spain); Julián Mauro Ramos Aceitero (Servicio Extremeño de Salud, Junta de Extremadura, Mérida, Spain).

\section{References}

1. Glass TA \& McAtee MJ (2006) Behavioral science at the crossroads in public health: extending horizons, envisioning the future. Soc Sci Med 62, 1650-1671.

2. Vitoria I \& Dalmau J (2003) Prevalencia de la obesidad en la infancia y la adolescencia. Actividades desde la atención primaria (Prevalence of obesity in childhood and adolescence. Activities from primary care). Pediátrika 23, 373-382.

3. Gobierno de España, Ministerio de Sanidad y Consumo (2007) $1^{a}$ Conferencia de Prevención y Promoción de la Salud en la Práctica Clinica en España. Prevención de la obesidad infantil y juvenil (1st Conference on Prevention and Health Promotion in Clinic Practice. Prevention of Childhood and Youth Obesity). Madrid: Ministerio de Sanidad y Consumo.

4. Santos JL, Ochoa MC, Patiño A et al. (2005) No evidence of association between the serotonin $2 \mathrm{~A}$ receptor $-1438 \mathrm{G} / \mathrm{A}$ promoter polymorphism and childhood obesity in a Spanish population: a case-parent study and a matched case-control study. Nutr Neurosci 8, 207-211.

5. Larqué E, Gil-Campos M, Ramírez-Tortosa MC et al. (2006) Postprandial response of trans fatty acids in prepubertal obese children. Int J Obes (Lond) 30, 1488-1493.

6. Ochoa MC, Santos JL, Azcona C et al. (2007) Association between obesity and insulin resistance with UCP2-UCP3 gene variants in Spanish children and adolescents. Mol Genet Metab 92, 351-358.

7. Ochoa MC, Moreno-Aliaga MJ, Martínez-González MA et al. (2007) Predictor factors for childhood obesity in a Spanish case-control study. Nutrition 23, 379-384.

8. Vega Alonso AT, Zurriaga Llorens O, Galmés Truyols A et al. (2006) Guía de principios y métodos de las redes centinelas (Guide to the principles and methods of health sentinel networks in Spain). Gac Sanit 20, Suppl. 3, S52-S60.

9. Sobradillo B, Aguirre A, Aresti U et al. (2004) Curvas y Tablas de Crecimiento (Estudios Longitudinal y Transversal) (Growth Curves and Tables (Longitudinal and CrossSectional Surveys)). Bilbao: Fundación Faustino Orbegozo Eizaguirre.

10. Generalitat Valenciana, Conselleria de Sanitat (2007) Encuesta de Salud de la Comunitat Valenciana (2005) (2005 Health Survey of Comunitat Valenciana). Valencia: Dirección General de Ordenación Evaluación e Investigación Sanitaria, Oficina del Plan de Salud, Conselleria de Sanitat.

11. Dapcich V, Salvador G, Ribas L et al. (2007) Guía de la Alimentación Saludable (Guide for Healthy Eating). Madrid: Sociedad Española de Nutrición Comunitaria.

12. Aranceta J, Pérez-Rodrigo C, Ribas L et al. (2003) Sociodemographic and lifestyle determinants of food patterns in Spanish children and adolescents: the enKid study. Eur J Clin Nutr 57, Suppl. 1, S40-S44.

13. Zurriaga O, Martínez-Beneito MA, Abellán JJ et al. (2004) Assessing the social class of children from parental information to study possible social inequalities in health outcomes. Ann Epidemiol 14, 378-384.

14. Agresti A (2002) Categorical Data Analysis, 2nd ed. New York: John Wiley \& Sons, Inc.

15. R Development Core Team (2009) R: A Language and Environment for Statistical Computing. Vienna: R Foundation for Statistical Computing.
16. Cole TJ, Bellizzi MC, Flegal KM et al. (2000) Establishing a standard definition for child overweight and obesity worldwide: international survey. BMJ 320, 1240-1243.

17. Cole TJ, Freeman JV \& Preece MA (1998) British 1990 growth reference centiles for weight, height, body mass index and head circumference fitted by maximum penalized likelihood. Stat Med 17, 407-429.

18. Moreno L, Sarriá A, Fleta J et al. (2000) Trends in body mass index and overweight among children and adolescents in the region of Aragon (Spain) from 1985 to 1995. Int J Obes Metab Disord 24, 925-931.

19. Livingstone B (2000) Epidemiology of childhood obesity in Europe. Eur J Pediatr 159, Suppl. 1, S14-S34.

20. Poskitt EME, and the European Childhood Obesity Group (1995) Committee report. Defining childhood obesity: the relative body mass index (BMI). Acta Paediatr 84, 961-963.

21. Serra-Majem L, Ribas-Barba L, Pérez-Rodrigo C et al. (2007) Methodological limitations in measuring childhood and adolescent obesity and overweight in epidemiological studies: does overweight fare better than obesity? Public Health Nutr 10, 1112-1120.

22. Hernández M, Castellet J, Narvaiza JL et al. (1988) Curvas y Tablas de Crecimiento (Growth Curves and Tables). Madrid: Editorial Garsi.

23. Daniels SR, Arnett DK, Eckel RH et al. (2005) Overweight in children and adolescents. Circulation 111, 1999-2012.

24. Gordon-Larsen P, Adair LS \& Popkin BM (2003) The relationship of ethnicity, socioeconomic factors, and overweight in US adolescents. Obes Res 11, 121-129.

25. Parsons TJ, Power C, Logan S et al. (1999) Childhood predictors of adult obesity: a systematic review. Int J Obes Relat Metab Disord 23, Suppl. 8, S1-S107.

26. Burke V, Beilin LJ, Simmer K et al. (2005) Predictors of body mass index and associations with cardiovascular risk factors in Australian children: a prospective cohort study. Int J Obes (Lond) 29, 15-23.

27. Gibson LY, Byrne SM, Davis EA et al. (2007) The role of family and maternal factors in childhood obesity. Med $J$ Aust 186, 591-595.

28. Nicklas TA, Baranowski T, Baranowski J et al. (2001) Family and child-care provider influences on preschool children's fruit, juice, and vegetable consumption. Nutr Rev 59, 224-235.

29. Epstein LH, Valoski A, Wing RR et al. (1990) Ten-year follow-up of behavioural, family-based treatment for obese children. JAMA 264, 2519-2523.

30. Reed DR \& Price RA (1998) Estimates of the heights and weights of family members: accuracy of informant reports. Int J Obes Relat Metab Disord 22, 827-835.

31. Gorber SC, Tremblay M, Moher D et al. (2007) A comparison of direct vs. self-report measures for assessing height, weight and body mass index: a systematic review. Obes Rev 8, 307-326.

32. Dauphinot V, Wolff H, Naudin F et al. (2009) New obesity body mass index threshold for self-reported data. J Epidemiol Community Health 63, 128-132.

33. Ezzati M, Martin H, Skjold S et al. (2006) Trends in national and state-level obesity in the USA after correction for selfreport bias: analysis of health surveys. J R Soc Med 99, 250-257.

34. Van den Bulck J \& Van Mierlo J (2004) Energy intake associated with television viewing in adolescents, a cross sectional study. Appetite 43, 181-184.

35. Vartanian LR, Schwartz MB \& Brownell KD (2007) Effects of soft drink consumption on nutrition and health: a systematic review and meta-analysis. Am J Public Health 97, 667-675.

36. Malik VS, Schulze MB \& Hu FB (2006) Intake of sugarsweetened beverages and weight gain: a systematic review. Am J Clin Nutr 84, 274-288.

37. Rodriguez-Artalejo F, López García E, Gorgojo L et al. (2003) Consumption of bakery products, sweetened soft 
drinks and yogurt among children aged 6-7 years: association with nutrient intake and overall diet quality. Br J Nutr 89, 419-428.

38. Bellisle F, McDevitt R \& Prentice AM (1997) Meal frequency and energy balance. Br J Nutr 77, Suppl. 1, S57-S70.

39. Toschke AM, Küchenhoff H, Koletzko B et al. (2005) Meal frequency and childhood obesity. Obes Res 13, 1932-1938.

40. Toschke AM, Thorsteinsdottir KH \& von Kries R (2009) Meal frequency, breakfast consumption and childhood obesity. Int J Pediatr Obes 4, 242-248.

41. Moreno LA, Rodríguez G, Fleta J et al. (2010) Trends of dietary habits in adolescents. Crit Rev Food Sci Nutr 50, 106-112.

42. Dubois L, Girard M \& Potvin Kent M (2006) Breakfast eating and overweight in a pre-school population: is there a link? Public Health Nutr 9, 436-442.

43. Moreno LA, Kersting M, de Henauw S et al. (2005) How to measure dietary intake and food habits in adolescence: the
European perspective. Int J Obes (Lond) 29, Suppl. 2, S66-S77.

44. Aranceta J, Pérez C, Ribas L et al. (2005) Epidemiología y factores determinantes de la obesidad infantil y juvenil en España (Epidemiology and determinants of childhood and adolescent obesity in Spain). Rev Pediatr Aten Primaria 7, Suppl. 1, S13-S20.

45. Vioque J, Weinbrenner T, Castello A et al. (2008) Intake of fruits and vegetables in relation to 10-year weight gain among Spanish adults. Obesity 16, 664-670.

46. Willett WC, Sacks F, Trichopoulou A et al. (1995) Mediterranean diet pyramid: a cultural model for healthy eating. Am J Clin Nutr 61, Suppl. 6, S1402-S1406.

47. Patel S \& Hu FB (2008) Short sleep duration and weight gain: a systematic review. Obesity (Silver Spring) 16, 643-653.

48. Cappuccio FP, Taggart FM, Kandala NB et al. (2008) Metaanalysis of short sleep duration and obesity in children and adults. Sleep 31, 619-626. 